Journal of Mathematics and Statistics 8 (2): 258-263, 2012

ISSN 1549-3644

(C) 2012 Science Publications

\title{
Mathematical Analysis of the Non Linear Epidemic Model
}

\author{
Laid Chahrazed-Rahmani Fouad Lazhar \\ Department of Mathematics, \\ Faculty of sciences Exactes, University Mentouri, Constantine, Algeria
}

\begin{abstract}
Classical epidemic models assume that the size of the total population is constant. More recent models consider a population size variable to take into account a longer period with death and disease causing reduced reproductive. The model contains a disease-free equilibrium and one or multiple equilibria are endemic. The stability of a disease-free status equilibrium and the existence of other nontrivial equilibria can be determined by the ratio called the basic reproductive number, which quantifies the number of secondary infections arise from a simple put infected in a population of sensitive. First, the local stability of the infection-free equilibrium and endemic equilibrium were analyzed, respectively. Second, the endemic equilibrium was formulated in terms of the incidence rate and local asymptotic stability. Finally we applied the adomian decomposition method to the system Epidemiologic. This method yields an analytical solution in terms of convergent infinite power series.
\end{abstract}

Key words: Adomian decomposition method, basic reproduction number, model epidemiology, locally asymptotically stability, global stability

\section{INTRODUCTION}

Classical epidemic models assume that the size of the total population is constant. More recent models consider a population size variable to take into account a longer period with death and disease causing reduced reproductive.

Generally, a model contains a disease-free equilibrium and one or multiple equilibria are endemic. The stability of a disease-free status equilibrium and the existence of other nontrivial equilibria can be determined by the ratio called the basic reproductive number, which quantifies the number of secondary infections arise from a simple put infected in a population of sensitive.

In this study, we discuss the equilibrium and stability of the model SIQS epidemic with a constant infectious period which is made. Most models used in studies of dynamics of epidemics have used a simple description of the process of the disease. A particular assumption is made that the time which individuals remain infectious can be described by an exponential distribution. This distribution corresponds to the assumption that the chances in a given time interval are independent of time since infection. In fact, infectious diseases increasing function. These conditions allow many differences a constant infectious period.

To solve the system with the Adomian decomposition method for this we use the references of (Ghasemia et al., 2007; Evansa and Raslan, 2005; Zedan and Al-Aidrous, 2009; Raslan, 2004; Pamuk, 2005; Mohyud-Din, 2010; Makarov and Dragunov, 2010).
Model equations: The system is described by equations which are defined as follows Eq. 1:

$$
\left\{\begin{array}{l}
\dot{S}(t)=\left(\mu+\gamma-\mu_{1}-\beta\right) S(t)-\alpha I(t)-k S(t) I(t)+v \\
\dot{I}(t)=\beta S(t)-\left(\mu_{2}+\gamma-\alpha\right) I(t)+k S(t) I(t)+\rho \\
\dot{Q}(t)=\gamma I(t)-\gamma S(t)-\mu_{3} Q(t)
\end{array}\right.
$$

$\mathrm{S}(\mathrm{t}), \mathrm{I}(\mathrm{t})$ and $\mathrm{Q}(\mathrm{t})$ denote the sizes of the population susceptible to disease and infectious members, quarantine members with the possibility of infection through temporary immunity, respectively. Where $S(t)$ $+\mathrm{I}(\mathrm{t})+\mathrm{Q}(\mathrm{t})=\mathrm{N}(\mathrm{t})$ denotes the population size at time $\mathrm{t}$; It is assumed that all new burns are susceptible. The positive constants $\mu_{1}, \mu_{2}$ and $\mu_{3}$ represent the death rates of susceptible, infectious and quarantine. The positive constants $\mu$ and $\gamma$ represent the birth rate (from incidence) of the population and the recovery rate of infection, respectively. The positive constants $\alpha, \beta$ is the average numbers of contacts infective for $S$ and I. The positive constant $\gamma$, is the number of transfer or conversion of infected people quarantined. $\mathrm{K}$ the rate of unknown persons infected with are detected by the system. $v, \rho$ the positive constants are the parameters of immigration. The initial condition of (1) is given as Eq. 2:

$$
\begin{aligned}
& S(\eta)=\Phi_{1}(\eta), I(\eta)=\Phi_{2}(\eta), Q(\eta) \\
& =\Phi_{3}(\eta),-\tau \leq \eta \leq 0,
\end{aligned}
$$


where, $\Phi=\left(\Phi_{1}, \Phi_{2}, \Phi_{3}\right)^{\mathrm{T}} \in \mathrm{C}$ such that $\mathrm{S}(\eta)=\Phi_{1}(\eta)$ $=\Phi_{1}(0) \geq 0, \mathrm{I}(\eta)=\Phi_{2}(\eta)=\Phi_{2}(0) \geq 0, \mathrm{Q}(\eta)=\Phi_{3}(\eta)$ $=\Phi_{3}(0) \geq 0$.

Let $C$ denote the Banach space $C\left([-\tau, 0], R^{3}\right)$ of continuous functions mapping the interval $[-\tau, 0]$ into $\mathrm{R}^{3}$. With a biological meaning, we further assume that $\Phi_{\mathrm{i}}(\eta)=\Phi_{\mathrm{i}}(0) \geq 0$ for $\mathrm{i}=1,2,3$.

Consider the system without the parameters of immigrations and study the stability of the system and since Q (t) does not appear explicitly in the first two equations of the system (1) with the initial condition in (2), Eq. 3:

$$
\left\{\begin{array}{l}
\dot{S}(t)=\left(\mu+\gamma-\mu_{1}-\beta\right) S(t)+\alpha I(t)-k S(t) I(t), \\
\dot{I}(t)=\beta S(t)-\left(\mu_{2}+\gamma+\alpha\right) I(t)+k S(t) I(t) .
\end{array}\right.
$$
Eq. 4:

With the initial condition in (2) which becomes

$$
\mathrm{S}(\eta)=\Phi_{1}(\eta), \mathrm{I}(\eta)=\Phi_{2}(\eta), \mathrm{Q}(\eta)
$$$$
=\Phi_{3}(\eta),-\tau \leq \eta \leq 0,
$$

where, $\Phi_{1}(0) \geq 0, \Phi_{2}(0) \geq 0, \Phi_{3}(0) \geq 0,-\tau \leq \eta \leq 0$ we obtain $\quad \dot{N}(\mathrm{t}) \leq \mu \mathrm{S}-\mu_{1} \mathrm{~N}(\mathrm{t})$ and $\mathrm{S}(\mathrm{t})+\mathrm{I}(\mathrm{t})+\mathrm{Q}(\mathrm{t}) \leq \mathrm{N}(\mathrm{t})$.

The region $\Omega=\left\{(\mathrm{S}, \mathrm{I}, \mathrm{Q}) \in \mathrm{R}^{3}+, \mathrm{S}+\mathrm{I}+\mathrm{Q} \leq \mathrm{N}\right.$ $\left.\prec \frac{\mu \mathrm{S}}{\mu_{1}}\right\}$ is positively invariant set of (3).

The disease free equilibrium and its stability: An equilibrium point of the system (1) satisfies Eq. 5:

$$
\left\{\begin{array}{l}
\left(\mu+\gamma-\mu_{1}-\beta\right) S(t)+\alpha I(t)-k S(t) I(t)=0 \\
\beta S(t)-\left(\mu_{2}+\gamma+\alpha\right) I(t)+k S(t) I(t)=0 .
\end{array}\right.
$$

We calculate the points of equilibria in the absence and presence of infection. In the absence of infection I $=0$, substituting in the system we obtain the first equilibrium point:

$$
\mathrm{O}_{0}=(\hat{\mathrm{S}}, \hat{\mathrm{I}})^{\mathrm{T}}=(0,0)^{\mathrm{T}}
$$

We calculate the Jacobian matrix according to the system (3) with $\mathrm{Q}_{0}$ :

$$
\mathrm{J}\left(\mathrm{Q}_{0}\right)=\left[\begin{array}{cc}
\mu+\gamma-\mu_{1}-\beta & \alpha \\
\beta & -\left(\mu_{2}+\gamma+\alpha\right)
\end{array}\right] .
$$

$\mathrm{Q}_{0}$, is locally asymptotically stable if and only if the trace of $\mathrm{J}\left(\mathrm{Q}_{0}\right)$ is strictly negative and its determinant is strictly positive.

$\mathrm{Q}_{0}$, is locally asymptotically stable Eq. 6:

$$
\Leftrightarrow\left\{\begin{array}{c}
\left(\mu-\mu_{1}-\mu_{2}-\alpha-\beta\right)-\mathrm{k}\left(\mathrm{S}^{*}+\mathrm{I}^{*}\right) \succ 0 \\
\left(\mu+\gamma-\mu_{1}\right)\left(\mu_{2}+\alpha+\beta\right)-\alpha \beta \succ 0
\end{array}\right.
$$

Endemic equilibrium and its locally asymptotically stability: We note that when the trivial equilibrium $\mathrm{Q}_{0}$ of system (3) is locally asymptotically stable, then the endemic equilibrium does not exist. In the presence of infection $I \neq 0$, substituting in the system (3) we obtain the second equilibrium point $Q^{*}=\left(S^{*} ; I^{*}\right)^{\mathrm{T}}$ Eq. 7:

$$
Q^{*}=\left(\begin{array}{l}
\frac{\mu_{2}+\alpha+\gamma}{\mathrm{k}}-\frac{\beta\left(\mu_{2}+\gamma\right)}{\mathrm{k}\left(\mu+\gamma-\mu_{1}\right)}, \frac{\mu+\gamma-\mu_{1}}{\mathrm{k}} \\
-\frac{\alpha\left(\mu+\gamma-\mu_{1}\right)}{\mathrm{k}\left(\mu_{2}+\gamma\right)}-\frac{\beta}{\mathrm{K}}
\end{array}\right)^{\mathrm{T}}
$$

Let $\mathrm{h}(\mathrm{t})=\mathrm{S}(\mathrm{t})-\mathrm{S}^{*}, \mathrm{~m}(\mathrm{t})=\mathrm{I}(\mathrm{t})-\mathrm{I}^{*} . \mathrm{h}$ and $\mathrm{m}$, is the small perturbations. We calculate the Jacobian matrix according to the system (3) with $Q^{*}$ :

$$
J\left(Q^{*}\right)=\left[\begin{array}{cc}
\left(\mu+\gamma-\mu_{1}-\beta\right)-k I^{*} & -\alpha \mathrm{k} S^{*} \\
\beta+k I^{*} & \mathrm{kS}^{*}-\left(\mu_{2}+\gamma+\alpha\right)
\end{array}\right] .
$$

$Q^{*}$; is locally asymptotically stable if and only if the trace of $\mathbf{J}\left(\mathrm{Q}^{*}\right)$ is strictly negative and its determinant is strictly positive. $\mathrm{Q}^{*}$, is locally asymptotically stable Eq. 8:

$$
\Leftrightarrow\left\{\begin{array}{c}
\left(\mu-\mu_{1}-\mu_{2}-\alpha-\beta\right)-\mathrm{k}\left(\mathrm{S}^{*}+\mathrm{I}^{*}\right) \succ 0 \\
\left(\mu+\gamma-\mu_{1}\right)\left(\mu_{2}+\alpha+\beta\right)-\alpha \beta \succ 0
\end{array}\right.
$$

The basic reproduction number $\mathbf{R}_{\mathbf{0}}$ : We calculate the Jacobian matrix at $\mathrm{Q}_{0}$ Eq. 9 and 10:

$$
\begin{array}{r}
\mathrm{KJ}\left(\mathrm{Q}_{0}\right)=\left(\begin{array}{cc}
\left(\mu+\gamma-\mu_{1}-\beta\right) & -\alpha \\
\beta & -\left(\mu_{2}+\gamma+\alpha\right)
\end{array}\right)=0 \\
\operatorname{det}\left(\begin{array}{cc}
\left(\mu+\gamma-\mu_{1}-\beta\right)-\lambda & -\alpha \\
\beta & -\left(\mu_{2}+\gamma+\alpha\right)-\lambda
\end{array}\right)=0
\end{array}
$$

So:

$$
\begin{aligned}
& \mathrm{P}(\lambda)=\lambda^{2}-\lambda\left(\mu-\mu_{1}-\mu_{2}\right. \\
& \mid-\alpha-\beta)-\left(\mu+\gamma-\mu_{1}-\beta\right)\left(\mu_{2}+\gamma+\alpha\right)+\alpha \beta
\end{aligned}
$$


We have traces of the matrix is negative, $\left(\mu-\mu_{1}-\mu_{2}-\alpha-\beta\right) \prec 0$. If $\operatorname{det}(\mathrm{J})>0$ then:

$$
-\left(\mu+\gamma+\mu_{1}-\beta\right)\left(\mu_{2}+\gamma+\alpha\right)+\alpha \beta \succ 0 .
$$
11:

The basic reproduction number $\mathrm{R}_{0}$ is defined as Eq.

$$
\mathrm{R}_{0}=\frac{\alpha}{\beta} \times \frac{\mu+\gamma+\mu_{1}}{\mu_{2}+\beta}+\frac{\mu+\gamma-\mu_{1}}{\beta}
$$

The basic reproduction number $\mathrm{R}_{0}$ is defined as the total number of infected population in the resulting subinfected population where almost all of the uninfected.

Theorem 1: The disease-free equilibrium $\mathrm{P}_{0}$ is locally asymptotically stable if $R_{0}<1$ and unstable if $R_{0}>1$.

The different parameters: It turns out that the stability of the endemic equilibrium is low with small perturbations are sufficient to maintain the recurrence of epidemics in the long term. On introduced a variation on the infectious contact rate, the rate is a variable used most is Eq. 12:

$$
\mathrm{a}(\mathrm{t})=\mathrm{a}_{0}\left(1+\mathrm{a}_{1} \cos (2 \pi \mathrm{t})\right)
$$

which $h \alpha_{0}$ is the average value of $\alpha$ and $\alpha_{1}$ and half the amplitude of the oscillations around this mean value.

The force of infection the key parameter of epidemiological model is the force of infection $\alpha$. This reflects the process of expressing of contamination an individual may contract the disease. It is this force of infection that puts individuals in the $\mathrm{S}$ compartment to compartment I. There are different ways to write this probability, two of they are density-dependent transmission of infection where the force is proportional. The number of patients in the host population Eq. 13:

$\mathrm{a}=\mathrm{ay}=\mathrm{a}_{0}\left(1+\mathrm{a}_{1} \cos (2 \pi \mathrm{t})\right)$

The transmission frequency dependence of the force of infection is proportional.

The proportion of patients in the population Eq. 14:

$\mathrm{a}=\frac{\mathrm{aI}}{\mathrm{S}+\mathrm{I}}=\frac{\mathrm{a}_{0}\left(1+\mathrm{a}_{1} \cos (2 \pi \mathrm{t})\right)}{\mathrm{S}+\mathrm{I}}$

Transmission density is directly dependent on the number of infected individuals. The frequencydependent transmission is common for the diseases directly transmitted for which the number of contacts is fixed. Indeed, for these diseases, the transmission is by close contact between a susceptible individual and an infected individual. However, the number of contacts is a fixed quantity, independent of the size of the total population and therefore the number of infected individuals.

\section{MATERIALS AND METHODS}

The Adomian decomposition method: The Adomian decomposition method has been applied to broad classes of problems in many fields such as mathematics, physics and biology. This method solves the functional equations of different types and the advantage of this method is that it solves a problem in the direct scheme, the solution is obtained as a series sounds fast converging. We consider the operator equation $\mathrm{Fu}=\mathrm{g}$, when $\mathrm{F}$ is the operator represents a general nonlinear ordinary differential and $G$ is a given function. The linear part of $F$ can be decomposed into $\mathrm{L}+\mathrm{R}, \mathrm{L}$ is easily invertible and $\mathrm{R}$ is the remainder of the F. It is therefore assumed that the nonlinear problem can be written as Eq. 15:

$\mathrm{Lz}+\mathrm{Rz}+\mathrm{Nz}=\mathrm{g}$

where, $\mathrm{N}$ represents the nonlinear terms $(\mathrm{N}$ is a nonlinear operator). $\mathrm{L}$ : is invertible ( $\mathrm{L}$ is the derivative highest for what is supposed to be invertible). $\mathrm{R}$ is a linear differential operator (of the order of less than L) and $g$ is the source term. We can be written Eq. 16:

$\mathrm{Lu}=\mathrm{g}-\mathrm{Ru}-\mathrm{Nu}$

Since $\mathrm{L}$ is invertible we also have Eq. 17:

$\mathrm{u}=\mathrm{a}+\mathrm{L}^{-1} \mathrm{~g}-\mathrm{L}^{-1}(\mathrm{Ru})-\mathrm{L}^{-1}(\mathrm{Nu})$

where, $\alpha$ is the solution of the homogeneous equation $\mathrm{Lu}=0$, with initial conditions. The decomposition of the nonlinear term $\mathrm{Nu}$ and to do so, Ado-mian developed a very elegant technique as follows. We define the parameter $\lambda$ decomposition, then $N(u)$ is a function of $\lambda, \mathrm{u}_{0}, \mathrm{u}_{1}, \ldots$, next expansion $\mathrm{N}(\mathrm{u})$ Maclurian series from $\lambda$.

We have $u$ in the form of a series Eq. 18:

$\mathrm{u}=\sum_{\mathrm{n}=0}^{\infty} \mathrm{u}_{\mathrm{n}}$

We decompose the nonlinear term, $\mathrm{N}$ as a series of special polynomials called Adomian polynomials Eq. 19:

$\mathrm{Nu}=\sum_{\mathrm{n}=0}^{\infty} A_{\mathrm{n}}$ 
These polynomials are obtained by introducing a parameter $\lambda$ and writing Eq. 20 :

$\mathrm{u}(\lambda)=\sum_{\mathrm{k}=0}^{\infty} \lambda^{\mathrm{k}}{ }_{\mathrm{uk}}$

We deduce that Eq. 21:

$\mathrm{A}_{\mathrm{n}}=\frac{1}{\mathrm{n} !}\left[\frac{\mathrm{d}^{\mathrm{n}}}{\mathrm{d} \lambda^{\mathrm{n}}} \mathrm{Nu}(\lambda)\right]_{\lambda=0}$

We have Eq. 22:

$\sum_{n=0}^{\infty} u_{n}=u_{0}-L^{-1} R \sum_{k=0}^{\infty} u_{n}-L^{-1} \sum_{n=0}^{\infty} A_{n}$

To determine the $u_{n}$ we can use the following method Eq. 23:

$$
\left\{\begin{array}{l}
\mathrm{u}_{0}=\mathrm{a}+\mathrm{L}^{-1} \mathrm{~g} \\
\mathrm{u}_{1}=-\mathrm{L}^{-1} \mathrm{Ru}_{0}-\mathrm{L}^{-1} \mathrm{~A}_{0} \\
\vdots \\
\mathrm{u}_{\mathrm{n}+1}=-\mathrm{L}^{-1} \mathrm{Ru}_{\mathrm{n}}-\mathrm{L}^{-1} \mathrm{~A}_{\mathrm{n}}
\end{array}\right.
$$

Then Eq. 24:

$u_{n}=-L^{-1} R\left(u_{n}-1\right)-L^{-1}\left(A_{n-1}\right), \quad N \geq 1$

Finally a solution is given as Eq. 25:

$\Phi_{N}(x)=\sum_{n=0}^{N-1} u_{n}(x), \quad N \geq 1$

The exact solution is Eq. 26:

$\mathrm{u}(\mathrm{x})=\lim _{\mathrm{N} \rightarrow \infty} \Phi_{\mathrm{N}}$

The resolution of the system with adomian decomposition method: It has a direct application of the Adomian decomposition method the system. We note that the system is a more general homogeneous system of ordinary differential equations where the nonlinear term is the product of two variables. We consider the general form of a system of differential equations given as follows Eq. 27:

$X_{i}^{\prime}=\sum_{j=1}^{n} a_{i j} X_{i j}+\sum_{p=1}^{n} \sum_{q=1}^{n} a_{i p q} X_{p} X_{q}, \quad i=1,2,3, \ldots, n$
We can write the system of equations above as an operator with $\mathrm{N}_{\mathrm{i}}$ is the first nonlinear term and I the second term is linear, $\mathrm{L}$ differential operator $\left(\frac{\mathrm{d}(.)}{\mathrm{dt}}\right)$ Eq. 28:

$\mathrm{LX}_{\mathrm{i}}=\mathrm{N}_{\mathrm{i}}+\mathrm{R}_{\mathrm{i}}, \quad \mathrm{i}=1,2,3, \ldots, \mathrm{n}$.

With applying the differential operator inverse $\mathrm{L}^{-1}$ we have Eq. 29:

$X_{i}(t)=X_{i}\left(t^{*}\right)+L^{-1} N_{i}+L^{-1} R_{i}, \quad i=1,2,3, \ldots, n$

The solution $\mathrm{X}_{\mathrm{i}}(\mathrm{t})$ is given as Eq. 30 :

$X_{i}(t)=\sum_{m=0}^{\infty} X_{i m}(t), \quad i=1,2,3, \ldots, n$

It was the first nonlinear term is Eq. 31:

$\mathrm{N}_{\mathrm{i}}=\sum_{\mathrm{j}=1}^{\mathrm{n}} \sum_{\mathrm{m}=0}^{\mathrm{n}} \mathrm{a}_{\mathrm{ij}} \mathrm{X}_{\mathrm{im}}$

With applying the differential operator inverse $\mathrm{L}^{-1}$ we have Eq. 32:

$L^{-1} N_{i}=\sum_{j=1}^{n} \sum_{m=0}^{\infty} a_{i j} \sum_{n=0}^{\infty} \int_{t^{*}}^{t} X_{i m} d t, \quad i=1,2,3, \ldots, n$.

It was the first linear term is Eq. 33:

$\mathrm{R}_{\mathrm{i}}=\sum_{\mathrm{p}=1}^{\mathrm{n}} \sum_{\mathrm{q}=0}^{\mathrm{n}} \mathrm{a}_{\mathrm{ipq}} \sum_{\mathrm{n}=0}^{\infty} \int_{\mathrm{t}^{*}}^{\mathrm{t}} \mathrm{M}_{\mathrm{im}, \mathrm{p}, \mathrm{q}}$

By applying the differential operator inverse $\mathrm{L}^{-1}$ sur l' Eq. 20 to obtain Eq. 34:

$\mathrm{L}^{-1} \mathrm{R}_{\mathrm{i}}=\sum_{\mathrm{p}=1}^{\mathrm{n}} \sum_{\mathrm{q}=0}^{\mathrm{n}} \mathrm{a}_{\mathrm{ipq}} \sum_{\mathrm{n}=0}^{\infty} \int_{\mathrm{t}^{*}}^{\mathrm{t}} \mathrm{M}_{\mathrm{im}, \mathrm{p}, \mathrm{q}} \mathrm{dt}$

For $\mathrm{i}=1,2,3, \ldots, \mathrm{n}$, we have Eq. 35 :

$\sum_{\mathrm{m}=0}^{\infty} \mathrm{X}_{\mathrm{im}}(\mathrm{t})=\mathrm{X}_{\mathrm{i}}\left(\mathrm{t}^{*}\right)+\sum_{\mathrm{j}=1}^{\mathrm{n}} \sum_{\mathrm{m}=1}^{\infty} \mathrm{a}_{\mathrm{ij}} \int_{\mathrm{t}^{*}}^{\mathrm{t}} \mathrm{X}_{\mathrm{im}} \mathrm{dt}$

$+\sum_{\mathrm{p}=1}^{\mathrm{n}} \sum_{\mathrm{q}=1}^{\mathrm{n}} \mathrm{a}_{\mathrm{ipq}} \int_{\mathrm{t}^{*}}^{\mathrm{t}} \mathrm{M}_{\mathrm{im}, \mathrm{p}, \mathrm{q}} \mathrm{dt}$

So if we write the solution for each $\mathrm{i}=1,2,3, \ldots, \mathrm{n}$. as follows Eq. 36-39:

$\mathrm{X}_{\mathrm{i} 0}(\mathrm{t})=\mathrm{X}_{\mathrm{i}}\left(\mathrm{t}^{*}\right)$ 


$$
\begin{aligned}
& X_{i 1}(t)=\sum_{j=1}^{n} a_{i j} \int_{t^{*}}^{t} X_{i 0} d t+\sum_{p=1}^{n} \sum_{q=1}^{n} a_{i p q} \int_{t^{*}}^{t} M_{i 0, p, q} d t \\
& X_{i 2}(t)=\sum_{j=1}^{n} a_{i j} \int_{t^{*}}^{t} X_{i 1} d t+\sum_{p=1}^{n} \sum_{q=1}^{n} a_{i p q} \int_{t^{*}}^{t} M_{i 1, p, q} d t \\
& X_{i,(m+1)}(t)=\sum_{j=1}^{n} a_{i j} \int_{t^{*}}^{t} X_{i, m} d t+\sum_{p=1}^{n} \sum_{q=1}^{n} a_{i p q} \int_{t^{*}}^{t} M_{i, m, p, q} d t
\end{aligned}
$$

By applying the Adomian polynomial and then the general solution, is defined as follows ( $\left.t-\mathrm{t}^{*}\right) \geq 0$ Eq. 40 :

$$
X_{i}(t)=\sum_{m=0}^{\infty} d_{i m} \frac{\left(t-t^{*}\right)^{m}}{m !}, \quad i=1,2,3, \ldots, n
$$

Solutions $\mathrm{d}_{\mathrm{im}}$ is given as follows Eq. 41and 42:

$$
\mathrm{d}_{\mathrm{i} 0}=\mathrm{X}_{\mathrm{i}}\left(\mathrm{t}^{*}\right)
$$

$\mathrm{d}_{\mathrm{im}}=\sum_{\mathrm{j}=1}^{\mathrm{n}} \mathrm{a}_{\mathrm{ij}} \mathrm{d}_{\mathrm{j}(\mathrm{m}-1)}+(\mathrm{m}-1) ! \sum_{\mathrm{p}=0}^{\mathrm{n}} \sum_{\mathrm{q}=0}^{\mathrm{n}} \sum_{\mathrm{k}=0}^{\mathrm{m}-1} \mathrm{a}_{\mathrm{ipq}} \frac{\mathrm{d}_{\mathrm{qk}}}{\mathrm{k} !}$

$\frac{d_{p(m-k-1)}}{k !(m-k-1) !} \quad m \geq 1$

Solve the system using the method (MADM): The solution explicite Eq. 43-45:

$$
\begin{aligned}
& \mathrm{S}=\sum_{\mathrm{m}=0}^{\infty} \mathrm{a}_{\mathrm{m}} \frac{\left(\mathrm{t}-\mathrm{t}^{*}\right)^{\mathrm{m}}}{\mathrm{m} !} \\
& \mathrm{I}=\sum_{\mathrm{m}=0}^{\infty} \mathrm{b}_{\mathrm{m}} \frac{\left(\mathrm{t}-\mathrm{t}^{*}\right)^{\mathrm{m}}}{\mathrm{m} !} \\
& \mathrm{Q}=\sum_{\mathrm{m}=0}^{\infty} \mathrm{c}_{\mathrm{m}} \frac{\left(\mathrm{t}-\mathrm{t}^{*}\right)^{\mathrm{m}}}{\mathrm{m} !}
\end{aligned}
$$

The coefficients are given to the relations occurrence as follows Eq. 46:

$\mathrm{a}_{0}=\mathrm{S}\left(\mathrm{t}^{*}\right), \quad \mathrm{b}_{0}=\mathrm{I}\left(\mathrm{t}^{*}\right), \quad \mathrm{c}_{0}=\mathrm{Q}\left(\mathrm{t}^{*}\right), \quad \mathrm{m} \geq 1$

We suppose that $\mathrm{N}=\mathrm{S}+\mathrm{I}+\mathrm{Q}$, then we have Eq. 47-49:

$$
\begin{aligned}
& \mathrm{a}_{\mathrm{m}}=\left(\mu+\lambda+\mu_{1}-\beta\right) \mathrm{a}_{(\mathrm{m}-1)}+\mathrm{ab}_{(\mathrm{m}-1)} \\
& -\mathrm{k}(\mathrm{m}-1) ! \sum_{\mathrm{k}=0}^{\mathrm{m}-1} \frac{\mathrm{a}_{\mathrm{k}} \mathrm{b}_{(\mathrm{m}-\mathrm{k}-1)}}{\mathrm{k} !(\mathrm{m}-\mathrm{k}-1) !}+\mathrm{v}
\end{aligned}
$$

$$
\begin{aligned}
& c_{\mathrm{m}}=\beta \mathrm{a}_{(\mathrm{m}-1)}+(\mathrm{m}-1) ! \sum_{\mathrm{k}=0}^{\mathrm{m}-1} \frac{\mathrm{a}_{\mathrm{k}} \mathrm{b}_{(\mathrm{m}-\mathrm{k}-1)} \mathrm{k} !(\mathrm{m}-\mathrm{k}-1) !}{-\left(\mu_{2}+\gamma+\mathrm{a}\right) \mathrm{b}_{(\mathrm{m}-1)}+\rho} \\
& \mathrm{c}_{\mathrm{m}}=\gamma \mathrm{b}(\mathrm{m}-1)-\mu_{3} \mathrm{c}(\mathrm{m}-1)-\gamma \mathrm{b}(\mathrm{m}-1)
\end{aligned}
$$

\section{RESULTS}

We study the model analytically SIQS constantly integrating the infectious period. Our results show that distributed for non-cyclic non-linear models of infectious diseases do not change the asymptotic behavior of local models. Sufficient conditions were given to ensure the existence of the endemic equilibrium for the system and the stability of the endemic equilibrium is studied, we have shown that under certain restrictions on the parameter values and the infectious period. The "endemic equilibrium is locally asymptotically stable, epidemiologically, this means that the disease will prevail and persist in a population.

\section{DISCUSSION}

From the values we have obtained that $\mathrm{k}$ is the parameter that varies the most relatively. The values of the equilibrium point $\mathrm{Q}^{*}$, are very sensitive to these variations, as $\mathrm{k}$ appears in the denominator of (7).

$\mathrm{O}_{0}$ end $\mathrm{Q}^{*}$, satisfied the conditions in (2) and (4) and they are locally asymptotically stable. The results obtained from the model give indications that any search method that is based on a group that has been in contact with persons that carry the virus is far more important in the control of the epidemic than a method, that is directed of the population.

\section{CONCLUSION}

In this study, we considered the stability of the few variable population SIQS epidemic models. We showed that if $\mathrm{R}_{0}<1$, the disease-free equilibrium is locally asymptotically stable, whereas if $R_{0}>1$, the endemic equilibrium is locally attractive. Then we resolve the system SIQS epidemic model with a delay with the method of decomposition adomian.

\section{REFERENCES}

Ghasemia, M., M.T. Kajanic and E. Babolian, 2007. Numerical solutions of the nonlinear integrodifferential equations: Wavelet-Galerkin method and homotopy perturbation method. Applied Math. Comput., 188: 450-455. DOI: 10.1016/j.amc.2006.10.001 
Evansa, D.J. and K.R. Raslan, 2005. The Adomian decomposition method for solving delay differential equation. Int. J. Comput. Math., 82: 4954. DOI: $10.1080 / 00207160412331286815$

Makarov, V. And D. Dragunov, 2010. A numericanalytical method for solving the Cauchy problem for ordinary differential equations. Cornell University Library.

Mohyud-Din, S.T., 2010. On numerical solutions of two-dimensional boussinesq equations by using adomian decomposition and he's homotopy perturbation method. Appli. Applied Math.: Int. J., 1: $1-11$.
Pamuk, S., 2005. An application for linear and nonlinear heat equations of Adomian's decomposition method. Applied Math. Comput., 163: 89-96. DOI: 10.1016/j.amc.2003.10.051

Raslan, K.R., 2004. The decomposition method for a Hirota-satsuma coupled $\mathrm{KdV}$ equation and a coupled MKdV equation. Int. J. Comput. Math., 81: 1497-1505. DOI: 10.1080/0020716042000261405

Zedan, H.A. and E. Al-Aidrous, 2009. Numerical solutions for a generalized Ito system by using Adomian decomposition method. Int. J. Math. Comput., 4: 9-19. 